\title{
Cluster: barriers of the central nervous system
}

\author{
Britta Engelhardt ${ }^{1}$
}

Published online: 3 February 2018

c) Springer-Verlag GmbH Germany, part of Springer Nature 2018

Cutting edge in vivo imaging technologies have become central in advancing our understanding of the structure and function of the central nervous system (CNS) in preclinical research and also in clinical diagnostics. Investigation of fluid movements and immune cell trafficking within the CNS in experimental animals by two-photon microscopy has challenged previous views on the movement of interstitial fluid (ISF) and cerebrospinal fluid (CSF) within the CNS [10] as well as the concept of the CNS immune privilege [11]. In the clinic, faster acquisitions and the development of advanced MRI sequences have improved the diagnosis of neurological disorders including analysis of brain barrier function. The intrinsic problem associated with any of the presently applied advanced preclinical and clinical imaging methodologies is that besides their limited resolution detection is limited to the molecular and cellular tracers applied. Thus, the correct interpretation of the video sequences recorded requires intimate knowledge of the anatomy of the CNS during health and its changes in disease and most importantly of the localization of the cellular and acellular barriers within the CNS, which may direct or limit the movement of the molecular or cellular tracers applied and imaged. Neglecting to properly consider the localization of brain barriers and how they may shape CNS functional compartments in the interpretation of advanced preclinical and clinical imaging sequences will invariably lead to misconceptions of CNS function.

The present Cluster on Barriers of the CNS, therefore, aims to provide a comprehensive overview on our current knowledge of the morphology, cellular and molecular characteristics and function of the brain barriers during health and disease. Historically, the endothelial blood-brain barrier (BBB) localized to the endothelial cells of CNS microvessels and the epithelial blood-cerebrospinal fluid barrier (BCSFB)

Britta Engelhardt

bengel@tki.unibe.ch

1 Theodor Kocher Institute, University of Bern, Bern, Switzerland localized in choroid plexus epithelium have long been in the focus of research in physiology and pharmacology and more recently also the fields of developmental biology, vascular biology and neuroimmunology. Thus, many excellent scholarly reviews have been published summarizing barrier characteristics of the BBB and BCSFB with either a focus on development or on their cellular junctions or on expression of transporters and efflux pumps in the context of drug delivery or in the context of their potential immune functions. Within this Cluster on Barriers of the CNS, we have rather aimed to provide a slightly different angle on BBB and BCSFB function by integrating barrier morphology and molecular anatomy as revealed by advanced imaging technologies with our recent understanding of the dynamic processes regulating these cellular barriers and how the specific barrier contributes to CNS homeostasis and disease. In their review entitled "Functional morphology of the blood-brain barrier in health and disease", Stefan Liebner and colleagues summarize our current understanding of the cellular and molecular mechanisms dynamically regulating BBB characteristics in CNS microvascular endothelial cells during development, health and CNS pathology. This includes in-depth considerations of the composition of the cellular and acellular components of the neurovascular unit (NVU) maintaining the BBB on one hand and how function of the BBB is impaired in CNS pathology as diverse as stroke, multiple sclerosis, brain tumors and Alzheimer's disease on the other hand. The authors also describe advanced imaging technology allowing to study BBB function and dysfunction and elaborate on our advanced understanding of the BBB as a regulator of CNS immune surveillance. The "Molecular anatomy and functions of the choroidal blood-cerebrospinal fluid barrier in health and disease" is addressed by Jean-Francois Ghersi-Egea and colleagues. This review summarizes current knowledge on the role of the choroid plexuses in brain development and homeostasis including CNS immune surveillance integrating advanced knowledge on the choroid plexus anatomy from the mesoscopic to the ultrastructural level. Furthermore, this review highlights the previously underestimated role of the choroid 
plexuses in maintaining the neural stem cell niche and in CNS pathology.

Although recent technological advances in the analysis of CNS functions by in vivo two-photon live cell imaging have literally "shed light" on the surface of the brain and spinal cord, they left the meningeal barriers "in the dark" due to the lack of appropriate molecular markers. The present lack of unequivocal identification of these important landmarks in two-photon imaging hampers precise spatial allocation of the fluorescently traced cellular or molecular interactions to the correct CNS compartment at the surface of the brain or spinal cord. In their review "The meninges as barriers and facilitators for the movement of fluid, cells and pathogens related to the rodent and human CNS" Roy Weller and colleagues describe the development of the meningeal layers and highlight their barrier functions during development and in the adult. The authors elaborate on the specific CNS compartments as established by the spatial arrangement of the meningeal barriers and layers and how these compartments allow for regulation of fluid flow and CNS immune surveillance without disturbing CNS homeostasis. In addition, they address how disturbances of the meningeal compartment in CNS pathologies affect CNS function. This review also accentuates that the dura mater lacks a BBB and thus naturally harbors lymphatic vessels, which have recently been re-discovered by employing novel lymphatic reporter mice and advanced imaging technologies [1]. These recent fluorescent tracer-based imaging studies have provided evidence for CSF drainage via dural lymphatic vessels to the brain draining deep cervical lymph nodes [12]. How CSF crosses the arachnoid barrier to reach the dural lymphatics has, however, not been solved. With claudin-11 and aquaporin 1 (AQP1), Weller and colleagues introduce novel molecular markers for the arachnoid barrier in their present review and demonstrate interruptions of the arachnoid barrier over the cribriform plate during human and rodent development. If maintained in adulthood this intriguing observation may explain, where a direct contact of CSF with dural lymphatics can occur. As dural lymphatics seem to cross the cribriform plate [1], these combined observations would support CSF drainage via the cribriform plate as previously described [4].

Finally, in the review "The role of brain barriers in fluid movement in the CNS-is there a "glymphatic" system?" Joan Abbott and colleagues aim to reconcile the established roles of the brain barriers in CNS fluid production and movement with the recently proposed concept of a "glymphatic system" (Fig. 1). The "glymphatic" concept is based on observations made on the distribution of fluorescent tracers or paramagnetic contrast agents applied to the CSF in experimental animals by two-photon imaging and dynamic contrast-enhanced MRI, respectively $[9,10]$. The findings have been interpreted such that there is a glial cell-dependent transparenchymal flow of subarachnoid CSF. The authors have then coined the term "glymphatic system" in analogy to the fluid clearance ensured by lymphatic vessels in peripheral organs. Based on our current knowledge, transparenchymal flow of CSF as proposed in the "glymphatic" concept is difficult to reconcile with the CNS immune privilege. While

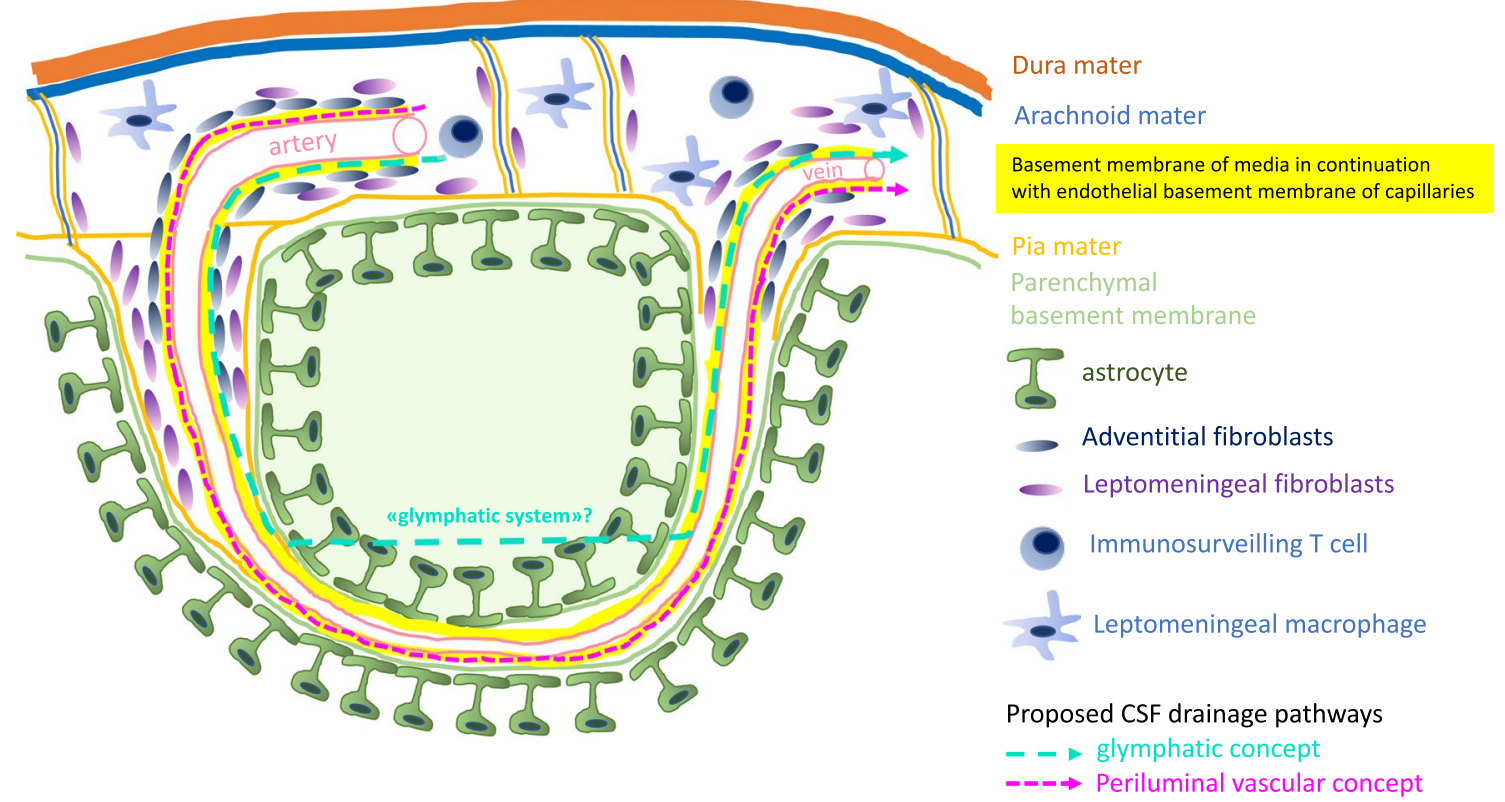

Fig. 1 Schematic representation of the leptomeningeal and perivascular spaces on the surface of the brain including a schematic outline of the proposed vascular periluminal CSF drainage pathways as pro-

posed by the "glymphatic system" or along an interconnected pathway of vascular basement membranes along the entire CNS vascular tree 
experimental introduction of foreign tissue transplants, bacterial factors or cytokines into the CNS parenchyma fails to elicit effective adaptive or innate immunity, this does not hold true, when the same triggers are introduced into the CSF-drained ventricular spaces (summarized in [7]). These observations underscore that the barriers of the CNS establish an architecture that allows the separation of the ISF-drained CNS parenchyma from the CSF-drained ventricular and leptomeningeal compartments, at least with respect to immune responses. This has previously led us to suggest that the CNS immune privilege is established by a brain barrier architecture resembling that of a medieval castle [5]. Using this analogy, the outer barriers, namely the BBB and BCSFB, strictly limit immune cell entry into the CSF filled "castle moat" to ensure CNS immune surveillance by allowing them to interact with antigen-presenting cells strategically localized in the subarachnoid or perivascular spaces. The glia limitans as the wall of the "moat" facing the "castle" maintains CNS homeostasis and is only breached by immune cells upon their local activation within the CSF-drained spaces during CNS pathologies leading to disturbances of CNS homeostasis and clinical disease [6].

Considering these apparently discrepant observations described above, it is important to ask whether the advanced imaging studies on the distribution of CSF applied tracers have to be interpreted differently. In this context, blood vessels and perivascular spaces have moved into the focus of attention, as the fluorescent tracers applied to the CSF were observed to rapidly drain along compartments surrounding the vascular lumen of leptomeningeal and of penetrating arteries [10]. Lacking precise anatomical markers, these compartments have been referred to as "perivascular" or "paravascular" compartments suggesting that drainage occurs around but outside of the vascular structure. The present review by Abbott and colleagues summarizes novel evidence for CSF drainage in the arterial media along the basement membranes between the smooth muscle cells, which are in direct continuation with the endothelial basement membrane of CNS capillaries and further on towards venules and veins (Fig. 1). The CNS may thus harbor a route of vascular "periluminal" CSF fluid flow along the extracellular matrix components within the blood vessel walls, which should thus be considered "intramural" rather than "perivascular" as it occurs within defined layers of the vascular wall. In fact, a recent study that followed CSF drainage of fluorescently labeled IgG by confocal microscopy found the fluorescent signal to accumulate within the arterial media and along the parenchymal basement membranes with no tracer detectable in between these defined layers [8]. These observations suggest that CSF may drain along intramural vascular extracellular matrix compartments and eventually along the parenchymal basement membrane of the glia limitans, which would allow for mixing with ISF at the level of microvascular basement membranes [2]. The limited resolution of two-photon imaging and dynamic contrast-enhanced MRI would not allow to distinguish CSF tracer distribution along those vascular and parenchymal basement membranes as compared to their transparenchymal flow as suggested by the "glymphatic" concept.

Last but not least, it is worthwhile to consider the role of the leptomeningeal and perivascular spaces in CNS fluid dynamics and immune surveillance of the CNS as touched upon to various degrees in the present reviews of the Cluster on the Barriers of the CNS. These spaces are by no means acellular fluid-filled spaces but rather harbor connective tissue and fibroblasts that may directly merge with the adventitia of arteries and veins. At least a subset of leptomeningeal fibroblasts, repeatedly referred to in the present reviews, expresses podoplanin and the extracellular matrix protein ERTR7 and thus resembles fibroblast reticular cells (FRCs), a major stromal cell population in lymph nodes involved in regulating fluid flow and CCR7-dependent lymphocyte homing by producing the CCR7 ligand CCL19. Employing a novel reporter mouse that allows to image CCL19-expressing cells, a recent study demonstrated that CNS infection triggers the expansion of a meningeal and perivascular network of CCL19-producing podoplanin ${ }^{+}$ERTR $7^{+}$FRC-like fibroblastic cells that are not detectable in the healthy CNS [3]. Thus, the fibroblastic stromal cells localized in the CSFdrained leptomeningeal and perivascular spaces generate confined microenvironments outside the CNS parenchyma that contribute to CNS immunity.

Bringing together interdisciplinary experts, the present Cluster on the Barriers of the CNS is meant to integrate our knowledge of the anatomy and function of the brain barriers with novel insights provided from novel reporter mouse models and advanced imaging technologies. The overall aim of this Cluster on the Barriers of the CNS is to trigger further discussion and novel research questions to advance our understanding of the role of the brain barriers in health and disease to ultimately help defining novel therapeutic targets for the treatment of the numerous devastating CNS pathologies by maintaining or reestablishing CNS barrier function.

Acknowledgements Dr. Urban Deutsch is gratefully acknowledged for critically reading this manuscript.

\section{References}

1. Aspelund A, Antila S, Proulx ST, Karlsen TV, Karaman S, Detmar M, Wiig H, Alitalo K (2015) A dural lymphatic vascular system that drains brain interstitial fluid and macromolecules. J Exp Med 212:991-999. https://doi.org/10.1084/jem.20142290

2. Carare RO, Hawkes CA, Weller RO (2014) Afferent and efferent immunological pathways of the brain. Anatomy, function and failure. Brain Behav Immun 36:9-14. https://doi.org/10.1016/j. bbi.2013.10.012 
3. Cupovic J, Onder L, Gil-Cruz C, Weiler E, Caviezel-Firner S, Perez-Shibayama C, Rulicke T, Bechmann I, Ludewig B (2016) Central nervous system stromal cells control local CD8(+) T cell responses during virus-induced neuroinflammation. Immunity 44:622-633. https://doi.org/10.1016/j.immuni.2015.12.022

4. Engelhardt B, Carare RO, Bechmann I, Flugel A, Laman JD, Weller RO (2016) Vascular, glial, and lymphatic immune gateways of the central nervous system. Acta Neuropathol 132:317338. https://doi.org/10.1007/s00401-016-1606-5

5. Engelhardt B, Coisne C (2011) Fluids and barriers of the CNS establish immune privilege by confining immune surveillance to a two-walled castle moat surrounding the CNS castle. Fluids Barriers CNS 8:4

6. Engelhardt B, Sorokin L (2009) The blood-brain and the bloodcerebrospinal fluid barriers: function and dysfunction. Semin Immunopathol 31:497-511. https://doi.org/10.1007/s0028 1-009-0177-0

7. Engelhardt B, Vajkoczy P, Weller RO (2017) The movers and shapers in immune privilege of the CNS. Nat Immunol 18:123131. https://doi.org/10.1038/ni.3666

8. Hannocks MJ, Pizzo ME, Huppert J, Deshpande T, Abbott NJ, Thorne RG, Sorokin L (2017) Molecular characterization of perivascular drainage pathways in the murine brain. J Cereb Blood Flow Metab. https://doi.org/10.1177/0271678x17749689

9. Iliff JJ, Lee H, Yu M, Feng T, Logan J, Nedergaard M, Benveniste H (2013) Brain-wide pathway for waste clearance captured by contrast-enhanced MRI. J Clin Invest 123:1299-1309. https://doi. org/10.1172/JCI67677

10. Iliff JJ, Wang M, Liao Y, Plogg BA, Peng W, Gundersen GA, Benveniste H, Vates GE, Deane R, Goldman SA et al (2012) A paravascular pathway facilitates CSF flow through the brain parenchyma and the clearance of interstitial solutes, including amyloid beta. Sci Transl Med 4:147ra111. https://doi.org/10.1126/ scitranslmed.3003748

11. Louveau A, Harris TH, Kipnis J (2015) Revisiting the mechanisms of CNS immune privilege. Trends Immunol 36:569-577. https:// doi.org/10.1016/j.it.2015.08.006

12. Ma Q, Ineichen BV, Detmar M, Proulx ST (2017) Outflow of cerebrospinal fluid is predominantly through lymphatic vessels and is reduced in aged mice. Nat Commun 8:1434. https://doi. org/10.1038/s41467-017-01484-6 